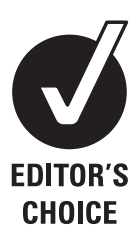

CHOICE

\title{
Probiotics and the immunological response to infant vaccinations: a prospective, placebo controlled pilot study
}

\author{
Ilan Youngster, ${ }^{1,2}$ Eran Kozer, ${ }^{1}$ Zipora Lazarovitch, ${ }^{3}$ Efrat Broide, ${ }^{4}$ Michael Goldman ${ }^{1}$
}

'Division of Paediatrics, Assaf Harofeh Medical Center. Zerifin, Israel

${ }^{2}$ Clinical Pharmacology Unit, Assaf Harofeh Medical Center, Zerifin, Israel

${ }^{3}$ Department of Microbiology, Assaf Harofeh Medical Center, Zerifin, Israel

${ }^{4}$ Gastroenterology Unit, Assaf Harofeh Medical Center, Zerifin, Israel

\section{Correspondence to}

Dr llan Youngster

Division of Paediatrics,

Assaf Harofeh Medical Center, Zerifin 70300, Israel; ilanyoungster@yahoo.com

Accepted 14 December 2010 Published Online First 24 January 2011

\begin{abstract}
Background Probiotics have been shown to be immunomodulatory and can affect antibody responses following vaccination. Several immunisations are associated with suboptimal seroconversion rates leaving a substantial part of the population exposed to infection. Objectives To evaluate the influence of probiotic supplementation on the immune response of infants following mumps, measles, rubella and varicella vaccination.

Methods A randomised, placebo-controlled, double blinded prospective trial was performed in a cohort of healthy infants. Study subjects were randomly assigned to receive probiotics or placebo for a total of 5 months, starting 2 months prior to vaccination. Antibody levels against vaccine components were measured 3 months after immunisation. Treatment-related and vaccinerelated adverse events were recorded.
\end{abstract}

Findings 47 infants completed the study, 25 in the probiotic group and 22 in the placebo group. There was no statistically significant difference in the number of infants failing to reach protective antibody titres against the different vaccine components (three infants in the placebo group against one in the treatment group for rubella, two each for mumps, four children vs two for measles). When combining all results in both groups, a larger percentage of failures to seroconvert occurred in the placebo group (17\% vs $8 \%, p=0.052)$, a result of borderline significance. The number of infants needed to treat in order to prevent one failed vaccine component was 12. There was no difference in the rate of treatment related adverse effects between the two groups. There was a significant trend toward fewer vaccine related adverse effects in the treatment group.

Conclusions Oral probiotics given to infants during the period of immunisation do not interfere with the immune response to mumps, measles, rubella and varicella vaccine, and may improve seroconversion rates.

\section{INTRODUCTION}

Immunisation is one of the most beneficial and cost-effective disease prevention measures. However, even in countries with maximal childhood immunisation coverage the protective effect is not optimal. For example, the efficacy of a single dose of varicella vaccine has been estimated in different studies to be between $70-85 \%$ against infection and $95 \%$ against severe disease, leaving a substantial proportion of children susceptible to infection. ${ }^{12}$

Several attempts have been made in the medical literature over the years to define the term 'probiotics', but the most complete definition was

\section{What is already known on this topic}

- Several immunisations are associated with suboptimal seroconversion rates, leaving part of the population exposed to infection.

- Probiotics have immunomodulatory properties influencing both the humoral and the cellular components of the immune system.

\section{What this study adds}

- Oral probiotics given to infants during the period of immunisation do not interfere with the immune response, and may improve seroconversion rates.

- Probiotics may potentially reduce vaccinerelated adverse effects in infants.

proposed in 2001 by Schrezenmeir and de Vrese: 'A preparation of or a product containing viable, defined microorganisms in sufficient numbers, which alter the microflora (by implantation or colonisation) in a compartment of the host and by that exert beneficial health effects in this host'. ${ }^{3}$ The precise mechanism of action of probiotics is not fully understood, but several animal and human studies have proven immunomodulatory effects involving both the humoral and the cellular components of the host's immune system. ${ }^{4-13}$ Klein et a ${ }^{14}$ demonstrated a significantly elevated percentage of granulocytes and monocytes showing phagocytic activity in a group of young adults treated with daily probiotics compared to placebo. Gill et al ${ }^{6}$ showed significantly enhanced serum antibody responses to orally and systemically administered antigens in a group of mice treated with daily probiotics. Several studies have shown an immune-enhancing effect following influenza vaccine in older people treated with probiotics ${ }^{15-18}$ and lately some authors have shown an enhanced antibody response in infants treated with probiotics following Haemophilus influenza b ( $\mathrm{Hib}$ ) and hepatitis B vaccinations. ${ }^{19-21}$

The main objective of the study was to evaluate whether supplementing the diet with oral probiotics enhances the immune response of infants following routine vaccination against mumps, measles, rubella and varicella (MMRV). Secondary outcomes included vaccine-related and treatmentrelated adverse effects. 


\section{PATIENTS AND METHODS \\ Study design}

The study was a prospective, randomised, parallel, double blind, controlled trial.

\section{Setting}

Study participants were recruited at the department of paediatrics, Assaf Harofeh Medical Center in Israel during January 2008 to August 2009. The study protocol was approved by the Institutional Review Board as well as by the National Ethics Committee in Paediatric Trials of the Israeli Ministry of Health (registered as ClinicalTrials.gov number, NCT 00645996).

\section{Study population}

All infants aged 8-10 months admitted to the paediatric ward with acute illness, whose guardians intended to follow the recommended immunisation schedule in Israel, were eligible for the study. Exclusion criteria were infants suffering from any chronic conditions resulting in immune depression, infants taking medications affecting the immune system, infants with permanent invasive catheters and those born prematurely (prior to gestational week 35).

\section{Supplementation}

The treatment group received a commercial formulation (Altman Probiotic Kid Powder; Altman, Maabarot, Israel) containing a combination of four different microorganisms: Lactobacillus acidophilus, strain ATCC4356, Bifidobacterium bifidum strain DSMZ20082, Bifidobacterium longum ATCC157078 and Bifidobacterium Infantis ATCC15697, with a concentration of $3 \times 10^{9}$ colony-forming units each. The control group received cornflour, matched in appearance and texture.

\section{Study protocol}

After obtaining written consent, the parents received a box containing 30 sachets of a daily dose of probiotics or placebo (figure 1). Participants were allocated to each group according to a computer generated randomisation list (six block randomisation). Investigators and the parents were blinded to the true content of the sachets. The guardian was asked to supplement the infant's daily intake with the content of a single sachet every day starting at age 10 months. At 12 months of age, children in Israel receive their first dose of live attenuated vaccine against MMRV (Priorix Tetra; GlaxoSmithKline,

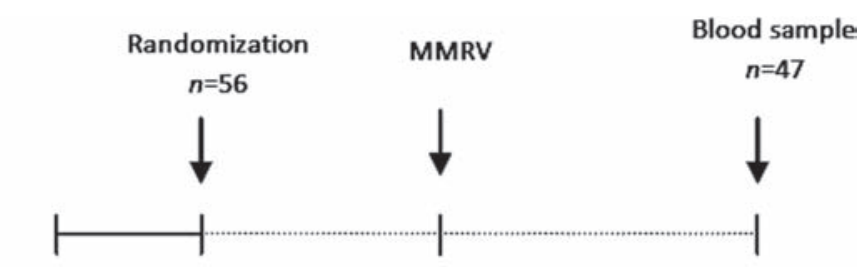

8 months 10 months

12 months

15 months

\section{Treatment period - probiotics/placebo}

Enrollement

Figure 1 Study flow chart. MMRV, mumps, measles, rubella and varicella.
Auckland, New Zealand). In addition, boosters are given for polio (inactivated polio vaccine), Hib and diphtheria-tetanuspertussis (DTP). The infants all received their vaccinations, as is customary, at the maternal and child welfare centres of the Israeli ministry of health. Three months after the vaccination a blood sample was collected from the infant and IgG levels against MMRV were measured. During this 5-month period the parents continued supplementation with probiotics/placebo. A research coordinator contacted the parents biweekly to ensure continuing compliance and to address any questions or concerns. During these calls information regarding untoward effects of the treatment was collected. Once monthly the supply of probiotics/placebo was replenished, and the empty sachets were collected, as another method of assessing compliance. Ten days following the immunisation a telephone interview was conducted and information was obtained regarding any untoward vaccine related adverse effects.

In Israel it is recommended by the Ministry of Health to screen all infants for anaemia around the age of 12 months by performing a complete blood count. We asked the parents to postpone the blood count for 3 months, and tested for vaccine responses at the same time, thereby avoiding exposing the infants to unnecessary painful procedures. The primary outcome of our study was the percentage of infants achieving protective antibody levels against vaccine components in the study group compared to the placebo group. Secondary outcomes included rates of vaccine-related and treatment-related adverse events in the two groups.

\section{Laboratory analysis}

The presence of protective levels of $\operatorname{Ig} G$ antibodies against vaccine components was determined by automated semiquantitative enzyme linked fluorescent assay according to the manufacturer's instructions. Vitek immunodiagnostic assay system (VIDAS) MSG, MPG, VZG and RBG were all analysed on a VIDAS automated immunoanalyser using a two-step enzyme immunoassay sandwich method (bioMerieux SA, Lyon, France). This system is more sensitive than conventional haemagglutination inhibition and indirect fluorescent antibody assays, and has high intra-assay reproducibility. Protective titres were determined by previous reports in the literature, ${ }^{12} 22$ and by the manufacturer's instructions. Optical density values were indexed according to the kit instructions corresponding to the following values: rubella $>40 \mathrm{IU} / \mathrm{ml}$, varicella $>150 \mathrm{mIU} / \mathrm{ml}$ and measles $>200 \mathrm{mIU} / \mathrm{ml}$. For mumps, as there is no known protective level of neutralising antibodies, a commonly used cut-off of $40 \mathrm{mIU} / \mathrm{ml}$ was used.

\section{Statistics}

Assuming a mean difference in the rate of seroconversion of $15 \%$ between the treatment and control groups, we estimated a required sample size of 63 participants in each group in order to achieve the required power $(85 \%$, type 1 error $=0.05$, two tailed test).

Numerical data are expressed using means \pm SDs and their medians and range. Categorical data are expressed using percentages. In order to compare between the two subgroups we used the test and the non-parametric Mann-Whitney test for two independent groups for the numerical data, and $\chi^{2}$ test and Fisher's exact test for the categorical data. The $p$ values for the comparison between the two groups are reported. All analyses were performed using SPSS V.14.0.1. 


\section{RESULTS}

During the trial period we approached 250 eligible participants, but succeeded in recruiting only 56 infants, of whom nine dropped out due to poor compliance (two subjects), refusal to immunise the child (two subjects), treatment-related adverse effects (two subjects with fussiness and abdominal pain, one in each group) and emigrating to another country (one subject). Two other participants gave no reason for the refusal to continue the study (figure 2). Baseline features of study participants were similar in both groups (table 1) and overall protective rates following vaccination were comparable with data from larger studies.

There was no significant difference between the two groups in the number of infants achieving protective titres of antibodies against each of the four vaccine components. (table 2). When we combined all the different antibody titres from each arm into a single group there was an advantage of borderline statistically significance to the treatment group, in achieving protective titres $(p=0.052)$.

Comparing the vaccine-related adverse events, as reported by the parents, there was a trend to fewer febrile episodes and fewer cases of diarrhoea in the treatment group (table 3), but the numbers were too small to reach statistical significance. When looking at the overall rate of adverse events there were fewer events in the treatment group $(p=0.051)$. Importantly, there was no difference in the rate of untoward side effects from treatment between the two groups.

\section{DISCUSSION}

To the best of our knowledge, this is the first study to evaluate the effect of probiotic supplementation on the antibody titres of infants following MMRV vaccination. Our study shows that probiotic supplementation does not interfere with the immune response of healthy infants to live vaccines. Moreover, we have shown a trend towards a better antibody responses in the probiotic treatment group, with more infants reaching protective titres 3 months after immunisation. This effect is modest, but can have an important impact especially when considering vaccines with suboptimal protective effects, such as varicella or flu. Interestingly, we found no difference in mean antibody concentration as determined by the semiquantitative fluorescence reading. This may suggest that when there is an adequate immune response, the probiotics have no additive effect on the quantity of antibodies produced, but

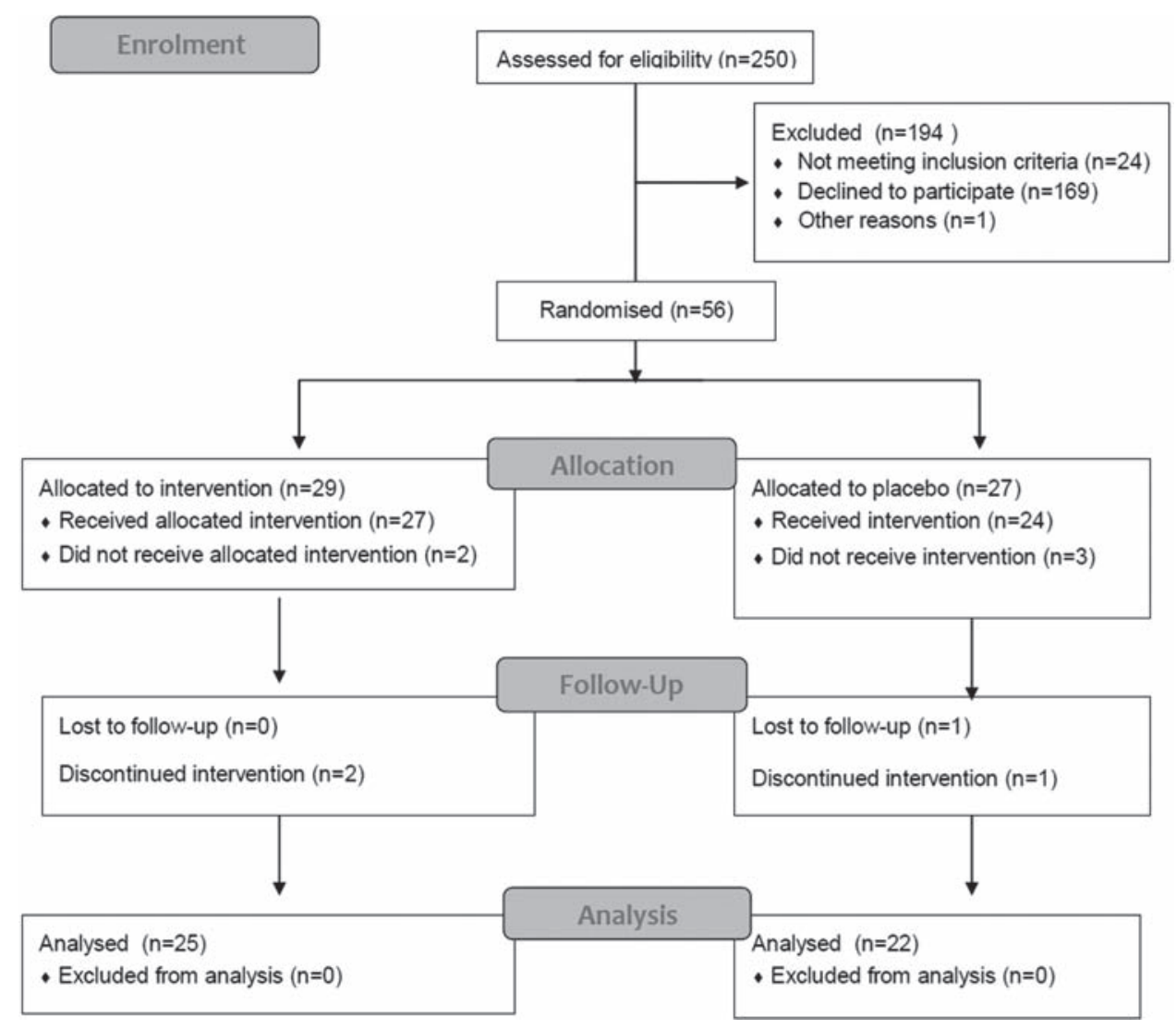

Figure 2 Revised CONSORT flow diagram.

Table 1 Comparison between study group variables

\begin{tabular}{llll}
\hline & Placebo group (n=22) & Probiotic group (n=25) & p Value \\
\hline Age when commencing treatment (months) & $9.5 \pm 0.9^{*}$ & $9.8 \pm 1.1^{*}$ & 0.56 \\
Age at time of vaccination (months) & $12.4 \pm 0.2^{*}$ & $12.5 \pm 0.3^{*}$ & 0.49 \\
Time between vaccination and blood sample (months) & $3.00(2.00,4.00) \dagger$ & $3.02(2.12,4.06) \dagger$ & 0.32 \\
Female/male & $12 / 10(57 \% / 42 \%)$ & $12 / 13(47 \% / 52 \%)$ & 0.54 \\
\hline
\end{tabular}

*Mean \pm SD.

†Median (minimum, maximum). 
Table 2 Number of study participants failing to reach protective IgG antibody titres 3 months after vaccination for mumps, measles, rubella and varicella

\begin{tabular}{lcll}
\hline & Placebo group & Probiotic group & p Value \\
\hline Rubella IgG & $3(13 \%)$ & $1(4 \%)$ & 0.226 \\
Mumps IgG & $2(9 \%)$ & $2(8 \%)$ & 0.392 \\
Measles IgG & $4(18 \%)$ & $2(8 \%)$ & 0.187 \\
Varicella IgG & $6(27 \%)$ & $3(12 \%)$ & 0.117 \\
All measured IgG antibodies combined & $15(17 \%)$ & $8(8 \%)$ & 0.052 \\
\hline
\end{tabular}

Table 3 Number of study participants reporting vaccine related adverse events up to 10 days following vaccination for mumps, measles, rubella and varicella as reported by the parents during phone interview

\begin{tabular}{lcll}
\hline & Placebo group & Probiotic group & p Value \\
\hline Fever & $5(22.7 \%)$ & $1(4.0 \%)$ & 0.072 \\
Restlessness & $4(18.1 \%)$ & $2(8.0 \%)$ & 0.286 \\
Diarrhoea & $2(9.0 \%)$ & $1(4.0 \%)$ & 0.484 \\
Any reported adverse effect & $11(16.6 \%)$ & $4(5.3 \%)$ & 0.051 \\
\hline
\end{tabular}

Fever: rectal temperature $>38.0^{\circ} \mathrm{C}$; restlessness was defined by the parents;

Diarrhoea: $>3$ loose stools per day.

that their value lies in a boosting effect only for infants with a weak immunological response to vaccination. Prior studies have shown that infants in developing countries usually mount higher antibody titres following vaccination, than do infants from developed countries. ${ }^{23}$ This has been explained by the 'hygiene hypothesis' stating that early repeated antigenic stimulation by viral and bacterial infections stimulates a Th1 profile of the immune response, favouring IgG2 production. Improvements in public health and hygiene, and the steady decline in family size in the developed world can thus potentially favour a Th2 response, explaining the reduced ability to mount adequate antibody responses following exposure to infections or vaccination. ${ }^{9} 1424$ Accordingly, Pérez et a ${ }^{23}$ showed recently that probiotic supplementation has no effect on antibody responses following DTP-Hib and 23-valent pneumococcal vaccine in children of low socioeconomic status in Argentina. It is possible that probiotic supplementation is a safe way of 'closing the gap' between infants in developed countries and those from developing parts of the world, regarding early microbial colonisation and antigenic stimulation.

Another finding in our current study was the trend to a reduced incidence of vaccine-related adverse events, mainly febrile episodes. This finding is especially important in light of the recent study by Prymula et al ${ }^{25}$ showing impaired antibody responses in infants who were treated with paracetamol following immunisation. If indeed our results are consistently reproduced in future studies, probiotic supplementation will have the added effect of potentially reducing the need for antipyretic administration after vaccination.

Consistent with the available literature, in our study there was no difference in the rate of reported adverse effects between the treatment groups among infants who completed the study. ${ }^{4} 26$ Only two subjects dropped out prematurely due to gastrointestinal adverse effects-one infant in each group. It appears that probiotic supplementation is a safe option for healthy infants.

There are several limitations to our study; first, the number of infants recruited was lower than expected. Our primary goal was to reach a cohort of 63 subjects in each group. After
2 years of recruitment we had to terminate the study without reaching our goal, and so even though there are several clear trends, most of our results are not statistically significant. In retrospect, statistical analysis of our results shows that if these trends persist, we would need 65 participants in each group, almost precisely matching our primary assumption. Nevertheless, we still feel our results are valuable, in that despite the sample size they show positive trends. This project is a pilot study and further investigations are needed.

One of the reasons we had difficulty recruiting children was that we enrolled healthy children, whereas most other studies published on the subject have enrolled children suffering from acute (diarrhoea or other infections) or chronic illnesses (prematurity, allergy), thus providing the parents with an incentive to participate. ${ }^{20} 2127-29$ In most of the clinical studies published on the influence of probiotics on immunisations, the children were part of a larger study examining the influence of probiotics on various conditions-mainly atopy. We feel that this is actually an important advantage of our study, as almost no other authors have shown immunomodulatory effects in healthy children.

Another common possible confounder is the question of compliance. In the current study we took measures to ensure compliance, and as our investigation was blinded and placebo controlled, even if there was some degree of non-compliance it would not have influenced the results.

Finally, previous studies have shown that not all probiotic preparations have the same beneficial effects. ${ }^{30}$ In our study we chose a common commercial preparation containing four different strains that have all been examined in previously published investigations, although not when given as a combination.

In conclusion, much has been written regarding the immunomodulatory effects of probiotic supplementations. Our current pilot study shows a trend towards higher seroconversion rates following vaccination with MMRV when infants are given daily probiotic supplementation. This effect is modest, and further studies are needed to elucidate the beneficial effects of probiotic preparations on the immune system of infants.

Acknowledgements This study was supported by a grant from the Materna infant nutrition research institute 2007.

Competing interests None.

Patient consent Obtained.

Ethics approval This study was conducted with the approval of the Institutional Review Board, Assaf Harofeh Medical Center, Israel and Ministry of Health Review Board, Israel.

Provenance and peer review Not commissioned; externally peer reviewed.

\section{REFERENCES}

1. Arbeter AM, Starr SE, Plotkin SA. Varicella vaccine studies in healthy children and adults. Pediatrics 1986;78:748-56.

2. Kuter BJ, Weibel RE, Guess HA, et al. Oka/Merck varicella vaccine in healthy children: final report of a 2-year efficacy study and 7-year follow-up studies. Vaccine 1991;9:643-7.

3. Schrezenmeir J, de Vrese M. Probiotics, prebiotics, and synbiotics approaching a definition. Am J Clin Nutr 2001;73:361-4S.

4. de Vrese M, Schrezenmeir J. Probiotics, prebiotics, and synbiotics. Adv Biochem Eng Biotechnol 2008;111:1-66.

5. Gill H, Prasad J. Probiotics, immunomodulation, and health benefits. Adv Exp Med Biol 2008;606:423-54.

6. Gill HS, Rutherfurd KJ, Prasad J, et al. Enhancement of natural and acquired immunity by Lactobacillus rhamnosus (HN001), Lactobacillus acidophilus (HN017) and Bifidobacterium lactis (HN019). Br J Nutr 2000;83:167-76.

7. Gill HS, Cross ML, Rutherfurd KJ, et al. Dietary probiotic supplementation to enhance cellular immunity in the elderly. Br J Biomed Sci 2001;58:94-6. 
8. Haghighi HR, Gong J, Gyles CL, et al. Probiotics stimulate production of natural antibodies in chickens. Clin Vaccine Immunol 2006;13:975-80.

9. Mohamadzadeh M, OIson S, Kalina WV, et al. Lactobacilli activate human dendritic cells that skew T cells toward T helper 1 polarization. Proc Natl Acad Sci USA 2005;102:2880-5.

10. Mohamadzadeh M, Klaenhammer TR. Specific Lactobacillus species differentially activate Toll-like receptors and downstream signals in dendritic cells. Expert Rev Vaccines 2008;7:1155-64.

11. Paineau D, Carcano D, Leyer G, et al. Effects of seven potential probiotic strains on specific immune responses in healthy adults: a double-blind, randomized, controlled trial. FEMS Immunol Med Microbiol 2008;53:107-13

12. Schierack P, Wieler LH, Taras D, et al. Bacillus cereus var. toyoi enhanced systemic immune response in piglets. Vet Immunol Immunopathol 2007;118:1-11.

13. Sheih YH, Chiang BL, Wang LH, et al. Systemic immunity-enhancing effects in healthy subjects following dietary consumption of the lactic acid bacterium Lactobacillus rhamnosus HN001. J Am Coll Nutr 2001:20:149-56.

14. Klein A, Friedrich U, Vogelsang H, et al. Lactobacillus acidophilus 74-2 and Bifidobacterium animalis subsp lactis DGCC 420 modulate unspecific cellular immune response in healthy adults. Eur J Clin Nutr 2008;62:584-93.

15. Boge T, Rémigy $M$, Vaudaine $S$, et al. A probiotic fermented dairy drink improves antibody response to influenza vaccination in the elderly in two randomised controlled trials. Vaccine 2009;27:5677-84

16. Bunout D, Hirsch S, Pía de la Maza M, et al. Effects of prebiotics on the immune response to vaccination in the elderly. JPEN J Parenter Enteral Nutr 2002:26:372-6.

17. de Vrese $\mathbf{M}$, Winkler $\mathrm{P}$, Rautenberg $\mathrm{P}$, et al. Probiotic bacteria reduced duration and severity but not the incidence of common cold episodes in a double blind, randomized, controlled trial. Vaccine 2006;24:6670-4.

18. Maeda N, Nakamura R, Hirose Y, et al. Oral administration of heat-killed Lactobacillus plantarum L-137 enhances protection against influenza virus infection by stimulation of type I interferon production in mice. Int Immunopharmacol 2009;9:1122-5.

19. Soh SE, Ong DQ, Gerez I, et al. Effect of probiotic supplementation in the first 6 months of life on specific antibody responses to infant Hepatitis B vaccination. Vaccine 2010;28:2577-9.
20. Taylor AL, Hale J, Wiltschut J, et al. Effects of probiotic supplementation for the first 6 months of life on allergen- and vaccine-specific immune responses. Clin Exp Allergy 2006;36:1227-35.

21. West CE, Gothefors L, Granström M, et al. Effects of feeding probiotics during weaning on infections and antibody responses to diphtheria, tetanus and Hib vaccines. Pediatr Allergy Immunol 2008;19:53-60.

22. Ercan TE, Soycan LY, Apak H, et al. Antibody titers and immune response to diphtheria-tetanus-pertussis and measles-mumps-rubella vaccination in children treated for acute lymphoblastic leukemia. J Pediatr Hematol Oncol 2005:27:273-7.

23. Pérez N, lannicelli JC, Girard-Bosch C, et al. Effect of probiotic supplementation on immunoglobulins, isoagglutinins and antibody response in children of low socio-economic status. Eur J Nutr 2010;49:173-9.

24. Mastrangeli G, Corinti S, Butteroni C, et al. Effects of live and inactivated VSL\#3 probiotic preparations in the modulation of in vitro and in vivo allergen-induced Th2 responses. Int Arch Allergy Immunol 2009;150:133-43.

25. Prymula R, Siegrist CA, Chlibek R, et al. Effect of prophylactic paracetamol administration at time of vaccination on febrile reactions and antibody responses in children: two open-label, randomised controlled trials. Lancet 2009;374:1339-50.

26. Gibson RA, Barclay D, Marshall $\mathrm{H}$, et al. Safety of supplementing infant formula with long-chain polyunsaturated fatty acids and Bifidobacterium lactis in term infants: a randomised controlled trial. Br J Nutr 2009;101:1706-13.

27. Kalliomäki M, Salminen S, Arvilommi H, et al. Probiotics in primary prevention of atopic disease: a randomised placebo-controlled trial. Lancet 2001;357:1076-9.

28. Kalliomäki M, Salminen S, Poussa T, et al. Probiotics and prevention of atopic disease: 4-year follow-up of a randomised placebo-controlled trial. Lancet 2003;361:1869-71.

29. Rosenfeldt V, Benfeldt E, Nielsen SD, et al. Effect of probiotic Lactobacillus strains in children with atopic dermatitis. J Allergy Clin Immunol 2003:111:389-95.

30. Canani RB, Cirillo P, Terrin G, et al. Probiotics for treatment of acute diarrhoea in children: randomised clinical trial of five different preparations. BMJ 2007;335:340. 